11,12

\title{
Фазовые переходы в низкоразмерных неупорядоченных моделях Поттса
}

\author{
(C) А.Б. Бабаев ${ }^{1,2}$, А.К. Муртазаев ${ }^{1}$ \\ ${ }^{1}$ Институт фризики им. Х.И. Амирханова ДФИЦ РАН, \\ Махачкала, Россия \\ ${ }^{2}$ Отдел математики и информатики ДФИЦ РАН, \\ Махачкала, Россия \\ E-mail: b_albert78@mail.ru
}

Поступила в Редакцию 30 декабря 2019 г.

В окончательной редакции 30 декабря 2019 г.

Принята к публикации 10 января 2020 г.

Методом Монте-Карло исследуются фазовые переходы в неупорядоченных двумерных моделях Поттса, в которых беспорядок реализован в виде немагнитных примесей. Получены температурные зависимости термодинамических параметров для теплоемкости $C$, восприимчивости $\chi$ и кумулянтов Биндера четвертого порядка в зависимости от концентрации спинов $p$. Расчеты проводились для систем с периодическими граничными условиями. Рассматривались системы с линейными размерами $L \times L=N, L=20-160$. Показано, что внесение в спиновую систему немагнитных примесей может привести к смене фазового перехода первого рода на фазовый переход второго рода. Обсуждается вопрос о классах универсальности критического поведения низкоразмерных разбавленных систем.

Ключевые слова: модель Поттса, примесь, фазовые переходы, критические индексы.

DOI: 10.21883/FTT.2020.05.49241.17M

\section{1. Введение}

Изучение влияния различных дефектов структуры, содержащихся в твердом теле в виде примесей или в виде дислокаций на фазовые переходы (ФП) и критические явления (КЯ) представляет большой теоретический и экспериментальный интерес $[1,2]$. Это связано с тем, что большинство реальных твердых тел всегда содержит примеси и другие дефекты структуры, присутствие которых влияет на их физические свойства и, в частности, может существенно влиять на поведение систем при ФП. По этой причине существует серьезная необходимость знать закономерности влияния примесей на те или иные свойства твердых тел. Без предварительных теоретических и экспериментальных исследований, ни один материал не может быть использован для практических целей. Поэтому в последнее время усилия многих исследователей были направлены на то, чтобы понять, как те или иные дефекты структуры влияют на поведение различных систем при ФП.

В работе [3] на основе перенормированной теории возмущений был выработан критерий Харриса, который позволяет на качественном уровне предсказать, в каких случаях примеси существенны для критического поведения, а в каких нет. Согласно этому критерию слабый беспорядок влияет на критическое поведение только в тех случаях, когда критический индекс теплоемкости положителен, $\alpha>0$, т. е. теплоемкость в точке перехода является расходящейся. В противоположном случае, когда $\alpha<0$, т. е. теплоемкость конечна в точке перехода, слабый беспорядок не влияет на критическое поведение.
Данному критерию удовлетворяют только системы, для которых эффективный гамильтониан которых вблизи критической точки изоморфен модели Изинга и $\alpha>0$. Критерий Харриса неприменим к двумерной модели Изинга в силу того, что $\alpha=0$. Детальное рассмотрение этого случая [4] позволило прийти к выводу, что влияние примеси затрагивает только поведение теплоемкости, в то время как остальные термодинамические и корреляционные функции не изменяют своего критического поведения. Исследованию критических свойств трехмерных неупорядоченных систем, описываемых моделью Изинга в последнее двадцатилетие было посвящено значительное число работ (см. $[5,6])$. Достигнут существенный прогресс в понимании особенностей влияния на эту модель вмороженного беспорядка.

С другой стороны, в случае спиновых систем, для которых в однородном состоянии наблюдается ФП первого рода примеси могут индуцировать ФП второго рода [7]. Для изучения этого случая наиболее хорошо подходят модели Поттса с числом состояний спина $q$. В моделях Поттса наблюдаются ФП первого рода при $q>4$, и ФП второго рода при $q \leq 4$. На этих моделях можно проверить особенности влияния примесей на ФП и определить их роль как стабилизирующего фактора при ФП второго рода. Кроме того, в соответствии с критерием Харриса в случае модели Поттса с $q=4$ примеси должны повлиять и на его критическое поведение, так как для этой модели $\alpha=2 / 3$.

В связи с этим целью настоящей работы является исследование на основе однокластерного алгоритма 
Вольфа метода Монте-Карло (МК) влияния слабого беспорядка, реализованного в виде вмороженных немагнитных примесей каноническим способом на ФП в низкоразмерных системах, для которых в однородном неразбавленном состоянии наблюдается ФП первого рода. Для этого рассматривались двумерная антиферромагнитная модель Поттса с числом состояний спина $q=3$ на треугольной решетке и ферромагнитная модель Поттса с $q=5$ на квадратной решетке. В случае неупорядоченной модели Поттса с $q=4$ исследовалось и критическое поведение.

\section{2. Описание модели и методика исследования}

В работе рассматриваются двумерные слабо разбавленные модели Поттса с числом состоянии спина $q=3$, $q=4$ и $q=5$. При построении модели Поттса необходимо иметь в виду следующие особенности: в узлах квадратной решетки расположены спины $\mathrm{Si}$, которые могут находиться в одном из q?2 состояний и немагнитные примеси (вакансии); немагнитные примеси распределены случайно и фиксированы (канонический способ) на различных узлах решетки (quenched disorder); энергия связи между двумя узлами равна нулю, если хотя бы в одном узле находится немагнитный атом, и равна , если взаимодействующие узлы находятся в одинаковых состояниях или если они находятся в разных состояниях (безразлично, в каких именно). С учетом этих особенностей микроскопический гамильтониан такой системы может быть, представлен в виде [8]

$$
H=\frac{1}{2} J \sum_{i, j} \rho_{i} \rho_{j} \cos \theta, \quad S_{i}=1,2,3,4,
$$

$\rho_{i}=\left\{\begin{array}{l}1, \text { если в узле расположен спин } \\ 0, \text { если в узле расопложена немагнитная примесь }\end{array}\right.$,

$\theta_{i, j}$ угол между взаимодействующими спинами $S_{i}-S_{j}$.

Расчеты проводились для систем с периодическими граничными условиями при концентрациях спинов $p=1.00,0.90$. Исследовались системы с линейными размерами $L \times L=N, L=20-160$. Начальные конфигурации задавались таким образом, чтобы все спины были упорядочены вдоль оси $Z$. Для вывода системы в равновесное состояние вычислялось время релаксации $\tau_{0}$ для всех систем с линейными размерами L. Этот неравновесный участок отбрасывали. Для самой большой системы $L=160, \tau_{0}=1.2 \cdot 10^{3} \mathrm{MK}$ steps/spin. Затем усреднение проводилось по участку марковской цепи длиной $\tau=300 \tau_{0}$. Кроме того, для повышения точности расчетов проводилось усреднение по 10 различным начальным конфигурациям. Затем эти данные использовались для расчета средних значений термодинамических параметров. Кроме, того для слабо разбавленных систем осуществлялось усреднение по 1000 различным конфигурациям распределения примесей в решетке.

\section{3. Результаты численного эксперимента}

Для наблюдения за температурным ходом поведения теплоемкости и восприимчивости использовались флуктуационные соотношения [9]:

$$
\begin{aligned}
& C=\left(N K^{2}\right)\left(\left\langle U^{2}\right\rangle-\langle U\rangle^{2}\right), \\
& \chi=(N K)\left(\left\langle m_{F}^{2}-\left\langle m_{F}\right\rangle^{2}\right),\right.
\end{aligned}
$$

где $K=|J| / k_{B} T, N=p L^{2}$ - число магнитных узлов, $U$ - внутренняя энергия, $m_{F}-$ намагниченность системы, угловые скобки обозначают усреднение по ансамблю. В качестве намагниченности $\left(m_{F}\right)$, для ФМ модели Поттса использовалось следующее выражение [10]

$$
m_{F}=\left\langle\frac{3}{2} \sum_{\alpha=1}^{5}\left(\frac{N_{\alpha}}{N}-\frac{1}{3}\right)^{2}\right\rangle^{1 / 2},
$$

где $N_{\alpha}=\left\{N_{1}, N_{2}, N_{3}, N_{4}, N_{5}\right\}, N_{1}$ - число спинов в состоянии с $q=1, N_{2}$ - число спинов в состоянии с $q=2, N_{3}$ - число спинов в состоянии с $q=3, N_{4}$ число спинов в состоянии с $q=4, N_{5}$ - число спинов в состоянии с $q=5$.

На рис. 1 и 2 представлены характерные зависимости теплоемкости $C$ и восприимчивости $\chi$ от температуры для модели Поттса с $q=4$ при концентрации магнитных узлов $p=1.0,0.9$. Здесь и далее погрешность данных не превышает размеры использованных символов на рисунках. Как видно из этих рисунков, наличие немагнитных примесей приводит к сглаживанию максимумов теплоемкости (рис. 1) и их уменьшению с ростом концентрации немагнитных атомов $c$, где $c=1-p$, что является характерной чертой для разбавленных систем [11-13]. Отметим также, что в критической области восприимчивость (рис. 2) имеет ярко выраженные максимумы при всех значениях концентраций $p$.

Для определения критических температур и анализа характера фазового перехода использовался метод кумулянтов Биндера четвертого порядка [14]:

$$
\begin{gathered}
V_{L}(T, p)=1-\frac{\left\langle E^{4}\right\rangle_{L}}{3\left\langle E^{2}\right\rangle_{L}^{2}}, \\
U_{L}(T, p)=1-\frac{\left\langle m^{4}(T, p ; L)\right\rangle_{L}}{3\left\langle m^{2}(T, p ; L)\right\rangle_{L}^{2}},
\end{gathered}
$$

где $E-$ энергия и $m-$ намагниченность системы с линейным размером $L$. Выражения (5) и (6) позволяют определить температуру фазового перехода $T_{l}(p)$ с большой точностью в фазовых переходах первого и второго рода соответственно. Методика определения температуры ФП этим методом рассмотрена в работах [15-18]. Следует отметить, что применение кумулянтов Биндера позволяет также хорошо тестировать род фазового перехода в системе. Известно, что фазовые 
переходы первого рода характеризуются следующими отличительными особенностями [19]: усредненная величина $V_{L}(T, p)$ стремится к некоторому нетривиальному значению $V^{*}$ согласно выражению

$$
V(T, p)=V^{*}+b L^{-d}
$$

при $L \rightarrow \infty$ и $T=T_{l}(L)$, где $V^{*}$ отлична от $2 / 3$, а минимальная величина $U_{L, \min }\left(T=T_{\min }, p\right)$ расходится $U_{L, \min }\left(T=T_{\min }, p\right) \rightarrow-\infty$ при $L \rightarrow \infty$, что и продемонстрировано на рис. 3 и 4 соответственно для исследованной нами модели Поттса с $q=5$ в отсутствии структурного беспорядка $(p=1.0)$; максимумы теплоемкости $C$ и восприимчивости $\chi$ пропорциональны объему $L^{d}$. Кроме того, в случае ФП второго рода кривые температурной зависимости кумулянтов Биндера $U_{L}(T, p)$ имеют четко выраженную точку пересечения. Характерные зависимости кумулянтов Биндера $V_{L}(T, p)$ и $U_{L}(T, p)$ от температуры для систем с разными линейными размерами при $p=0.90$ приведены на рис. 5 и 6 соответственно.

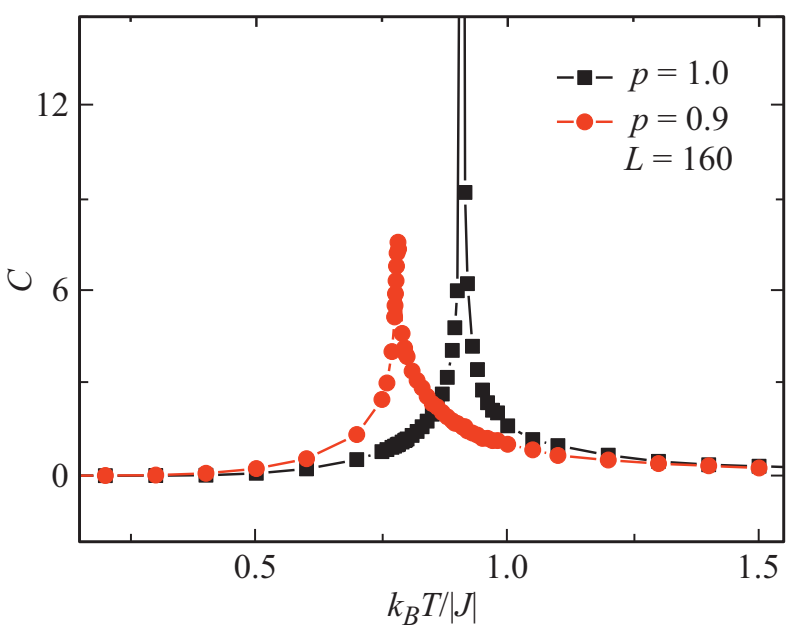

Рис. 1. Температурная зависимость теплоемкости $C$ для двумерной разбавленной модели Поттса с $q=4$.

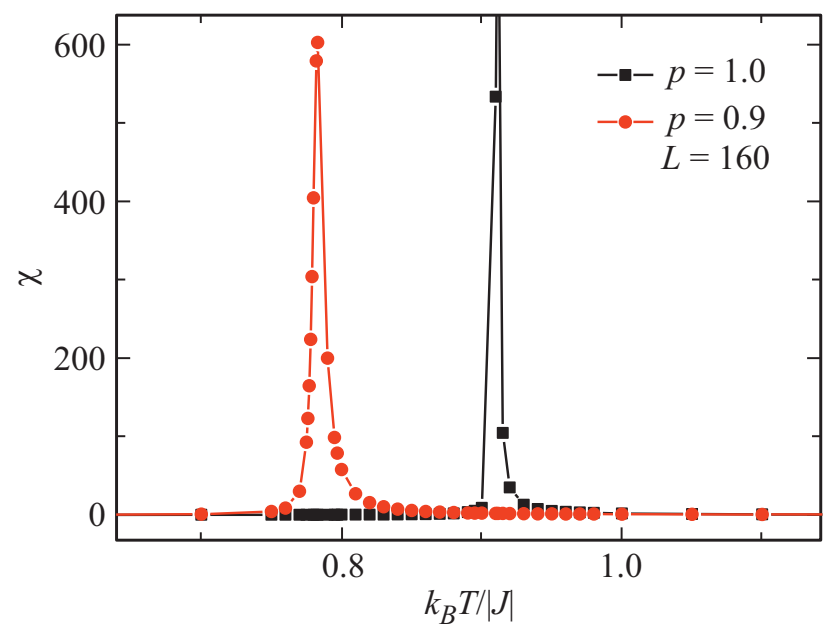

Рис. 2. Температурная зависимость восприимчивости $\chi$ для двумерной разбавленной модели Поттса с $q=4$.

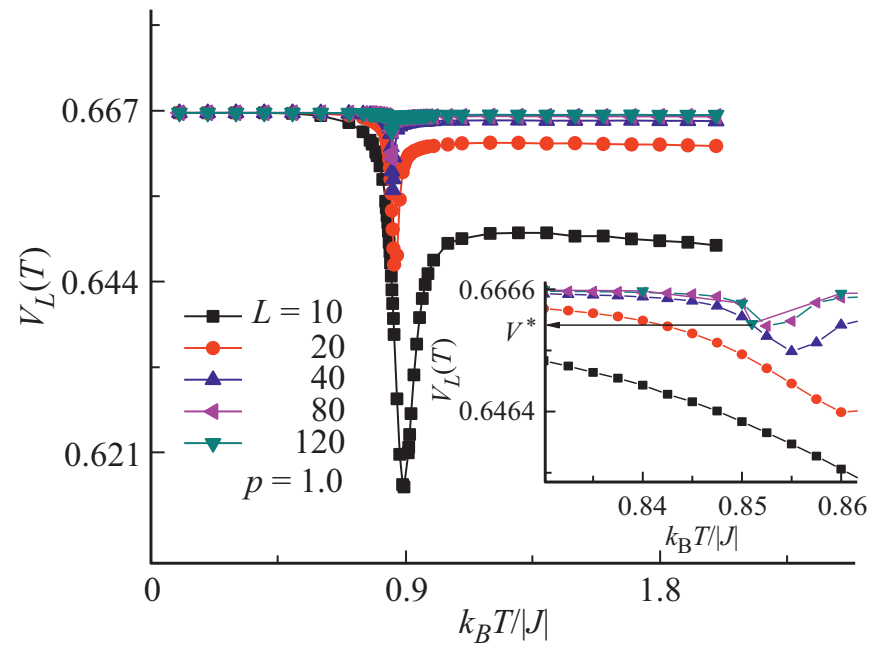

Рис. 3. Зависимость кумулянтов $V_{L}$ от температуры для чистой модели Поттса с $q=5$ при $p=1.0$.

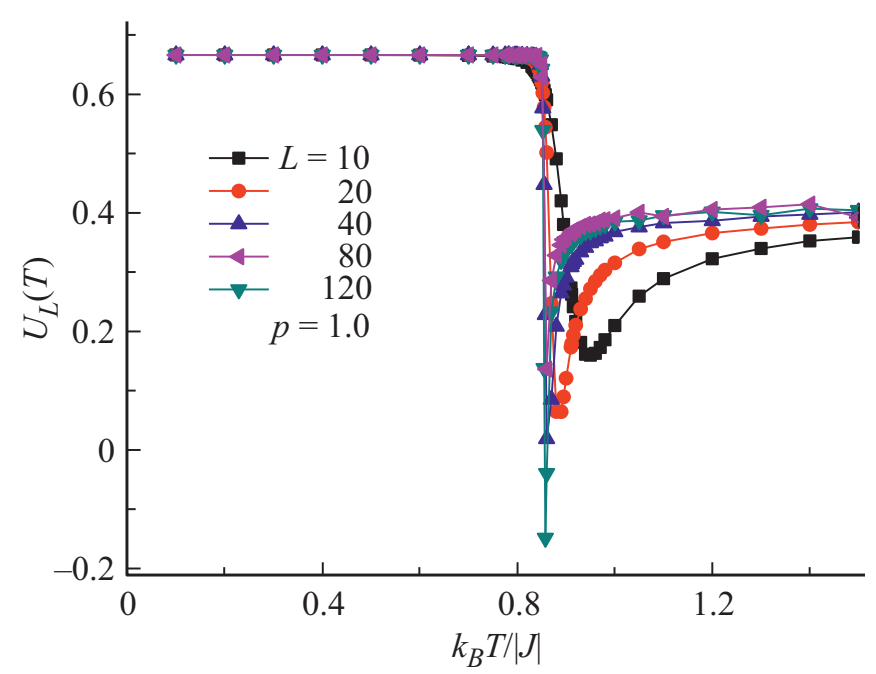

Рис. 4. Зависимость кумулянтов $U_{L}$ от температуры для чистой модели Поттса с $q=5$ при $p=1.0$.

Заметим, что из вставки к рисунку 5 наглядно видно, что нетривиальная величина $V^{*} \rightarrow 2 / 3$ в соответствии с выражением (7) при $L \rightarrow \infty$. Такое поведение как отмечалось выше характерно для ФП второго рода. Кроме того, из рис. 6 видно, что в критической области для $U_{L}(T, p)$ наблюдается четко выраженная точка пересечения и $U_{L}(T, p)$ не проявляет тенденцию стремления к $-\infty$ при $L \rightarrow \infty$, что также свидетельствует о ФП второго рода. Определенные методом кумулянтов Биндера температуры фазовых переходов $T_{l}(p)$ в единицах $|J| / k_{B}$ равны: $T_{l}(1.0)=0.8515(1), T_{l}(0.90)=0.731(2)$. Как видно, температура ФП полученная для чистой спиновой системы при $p=1.0$ достаточно хорошо согласуется с аналитическим значением полученным Бакстером [20] по формуле $\frac{k_{B} T_{l}}{|J|}=\frac{1}{\ln (1+\sqrt{5})}=0.8515 \ldots$ Для антиферромагнитной модели Поттса с $q=3$ на треугольной решетке 


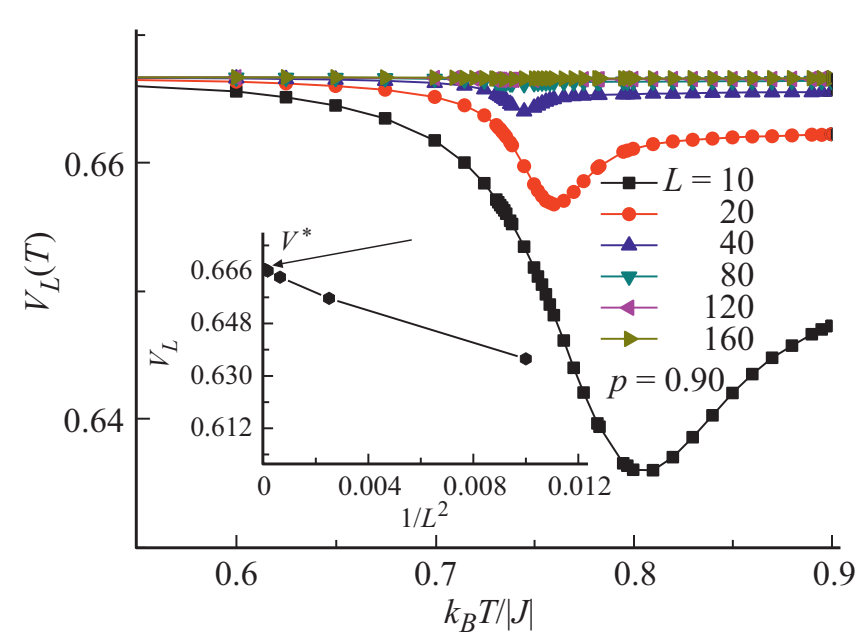

Рис. 5. Зависимость кумулянтов $V_{L}$ от температуры для слабо разбавленной модели Поттса с $q=5$ при $p=0.9$.

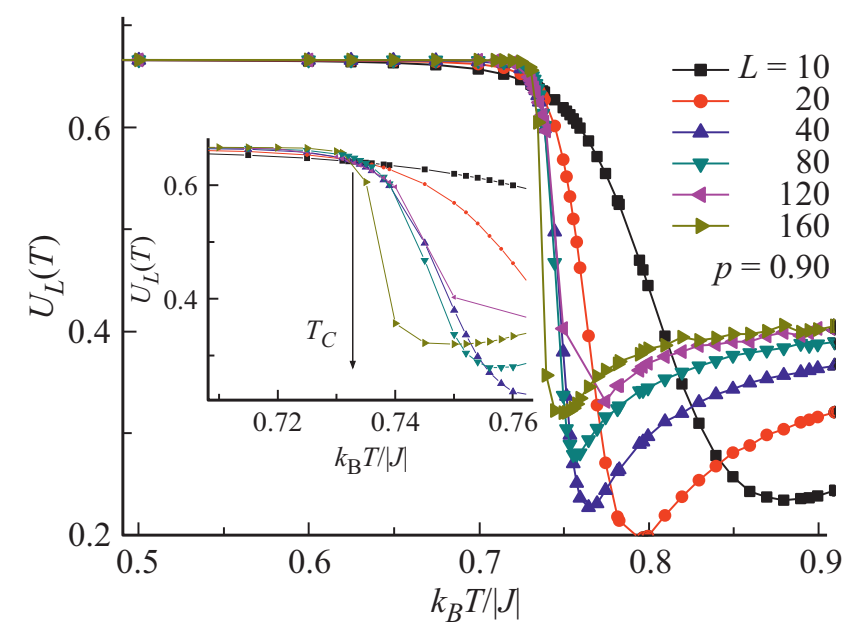

Рис. 6. Зависимость кумулянтов $U_{L}$ от температуры для слабо разбавленной модели Поттса с $q=5$ при $p=0.9$.

критические температуры разбавленных систем $T_{c}(p)$ в единицах $|J| / k_{B}$ были определены в работах $[12,21]$ и равны: $\quad T_{c}(0.90)=0.79(1), \quad T_{c}(0.80)=0.65(2)$, $T_{c}(0.70)=0.42(3), T_{c}(0.65)=0.35(4)$.

Для ферромагнитной модели Поттса с числом состояний спина $q=4$ на квадратной решетке как нами ранее было отмечено внесение примесей в соответствии с критерием Харриса должно сказаться на критическое поведение. Температуры фазовых переходов $T_{l}(p)$ в единицах $|J| / k_{B}$ для этой модели равны: $T_{l}(1.0)=0.910(1), T_{l}(0.90)=0.777(2)$. Для определения статических критических индексов (КИ) теплоемкости $\alpha$, восприимчивости $\gamma$ и намагниченности $\beta$ использовалась теория конечно-размерного скейлинга [22,23]. Методика определения КИ этим методом на примере антиферромагнитной неупорядоченной модели Поттса с $q=3$ на треугольной решетке подробно приведена в работах $[12,21]$. Для расчета критических индексов $\alpha, \beta$, $\gamma$ и $v$ строились зависимости $C, m, \chi$, и параметра $V_{n}$ от $L$. Анализ данных, выполненный с использованием нелинейного метода наименьших квадратов, позволил определить значения $\alpha / v, \beta / v, \gamma / v$ и $1 / v$. Затем, используя значения $v$, полученные в рамках данного исследования, определялись $\alpha, \beta$ и $\gamma$. Следует отметить, что во многих численных исследованиях неупорядоченных систем, в отличие от наших эти индексы в основном определялись, используя критический индекс $v$ полученный на основе различных скейлинговых соотношений.

Анализ данных, выполненный с использованием нелинейного метода наименьших квадратов, позволил определить значения $\alpha=0.665(1), \beta=0.083(1)$, $\gamma=1.165(1), \quad v=0.664(1)$ для однородной системы $(p=1.0)$ и $\alpha=0.67(1), \beta=0.09(1), \gamma=1.17(1)$, $v=0.67(1)$ для слабо разбавленной системы $(p=0.90)$. Полученные критические индексы в пределах погрешности удовлетворяют соотношениям теории конечноразмерного скейлинга и внесение небольшого беспорядка в виде немагнитных примесей приводит к незначительному их изменению.

Расчет критических индексов для неупорядоченной модели Поттса с $q=5$ требует отдельного рассмотрения. Определение КИ в широком диапазоне изменений концентраций немагнитных примесей $c, c=1-p$ позволит ответить на принципиальный вопрос об универсальности критического поведения разбавленных систем.

\section{4. Заключение}

В настоящей работе с соблюдением единой методики исследовано влияние слабого вмороженного беспорядка, реализованного в виде вмороженных немагнитных примесей на фазовые переходы в двумерных моделях Поттса на квадратной решетке. Данные полученные в результате наших исследований свидетельствуют о том, что:

1. В двумерной антиферромагнитной модели Поттса с $q=3$ на треугольной решетке и в ферромагнитной модели Поттса с $q=5$ на квадратной решетке наблюдаются фазовые переходы первого рода в соответствии с теорией среднего поля [8]. Внесение слабого беспорядка $(p=0.90)$ в виде вмороженных немагнитных примесей в рассматриваемых моделях приводят к ФП второго рода.

2. Критическое поведение неупорядоченной модели Поттса с $q=4$ описывается новым универсальным набором критических индексов.

\section{Финансирование работы}

Исследование выполнено при финансовой поддержке РФФИ в рамках научного проекта № 19-02-00153.

\section{Конфликт интересов}

Авторы заявляют, что у них нет конфликта интересов. 


\section{Список литературы}

[1] O. Vasilyev, B. Berche, M. Dudka, Yu. Holovatch. Phys. Rev. E 92, 042118 (2015).

[2] В.В. Прудников, А.Н. Вакилов, П.В. Прудников. Фазовые переходы и методы их компьютерного моделирования. ФИЗМАТЛИТ, М. (2009). 224 с.

[3] A.B. Harris. J. Phys. C 7, 1671 (1974).

[4] Vik. Dotsenko, Vl. Dotsenko. Adv. Phys. 32, 129 (1983).

[5] Р. Фольк, Ю. Головач, Т. Яворский. УФН 173, 175 (2003).

[6] А.Б. Бабаев, А.К. Муртазаев. Мат. моделирование 30, 12, 55 (2018).

[7] Y. Imry, M. Wortis. Phys. Rev. B 19, 3580 (1979).

[8] F.Y. Wu. ExactlySolved Models: A Journey in Statistical Mechanics. World Scientific, London (2009).

[9] P. Peczac, A.M. Ferrenberg, D.P. Landau. Phys. Rev. B 43, 6087 (1991).

[10] C. Chatelain, B. Berche. Phys. Rev. Lett. 80,1670 (1998).

[11] A.B. Babaev, A.K. Murtazaev. Low Temp. Phys. 44, 12, 1348 (2018).

[12] A.B. Babaev, A.K. Murtazaev. JETP Lett. 107, 10, 624 (2018).

[13] A.K. Murtazaev, A.B. Babaev, G.Ya. Ataeva. Phys. Solid State 59, 1, 141 (2017).

[14] K. Eichhorn, K. Binder. J. Phys.: Condens. Matter 8, 5209 (1996).

[15] A.B. Babaev, A.K. Murtazaev. Low Temp. Phys. 41, 8, 608 (2015).

[16] A.B. Babaev, T.R. Rizvanova, A.K. Murtazaev. Phys. Solid State 59,12, 2444 (2017).

[17] A.K. Murtazaev, A.B. Babaev, M.A. Magomedov, F.A. Kassan-Ogly, A.I. Proshkin. JETP Lett. 100, 242 (2014).

[18] A.B. Babaev, A.K. Murtazaev. Phys. Solid State 61, 7, 1284 (2019).

[19] D. Loison, K.D. Schotte. Europ. Phys. J. B 5, 735 (1998).

[20] Р. Бэкстер. Точно решаемые модели в статистической механике. Мир, М. (1985).

[21] A.K. Murtazaev, A.B. Babaev. Mater. Lett. 238, 321 (2019).

[22] M.E. Fisher, M.N. Barber. Phys. Rev. Lett. 28, 1516 (1972).

[23] D. Loison. Phys. Lett. A 257, 83 (1999).

Редактор Д.В. Жуманов 